\title{
An Evaluation of the Usefulness of Case-Based Explanation
}

\author{
Pádraig Cunningham, Dónal Doyle, John Loughrey \\ Department of Computer Science \\ Trinity College Dublin \\ Padraig.Cunningham@cs.tcd.ie
}

\begin{abstract}
One of the perceived benefits of Case-Based Reasoning (CBR) is the potential to use retrieved cases to explain predictions. Surprisingly, this aspect of CBR has not been much researched. There has been some early work on knowledge-intensive approaches to $\mathrm{CBR}$ where the cases contain explanation patterns (e.g. SWALE). However, a more knowledge-light approach where the case similarity is the basis for explanation has received little attention. To explore this, we have developed a CBR system for predicting blood-alcohol level. We compare explanations of predictions produced with this system with alternative rule-based explanations. The casebased explanations fare very well in this evaluation and score significantly better than the rule-based alternative.
\end{abstract}

\section{Introduction}

Most tutorials on Case-Based Reasoning (CBR) would point to the advantages arising from the transparency and interpretability of the CBR approach. This transparency has particular advantages for explanation as pointed out by Leake (1996):

“...neural network systems cannot provide explanations of their decisions and rule-based systems must explain their decisions by reference to their rules, which the user may not fully understand or accept. On the other hand, the results of CBR systems are based on actual prior cases that can be presented to the user to provide compelling support for the system's conclusions."

Given this potential for explanation it is perhaps surprising that explanation is not more prominent in CBR applications and is not a bigger issue in CBR research and development. This is particularly remarkable since the rise of data mining as an application area for Machine Learning (ML) techniques has raised the emphasis on interpretability and explanation in ML research generally.

In this paper we question the usefulness of case-based explanation (CBE) to users of decision support systems. Are explanations based on specific examples as useful as ones based on general principles? Although we are interested in this question from the perspective of medical decision support systems we have developed an alternative domain for the evaluation. We have developed a case-based Breathalyser 
system that will predict whether a subject is over the drink-driving limit based on a case-like description of the subject (see Fig. 1). We have developed this application because of the ready availability of subjects with some knowledge of the domain who can provide feedback on the explanations. It would be very difficult to get the same volume of feedback from medical practitioners in a specialised domain.

Before describing the evaluation we have performed, we review existing research on explanation in CBR in section 2. In this evaluation we describe two distinct approaches, the knowledge-light approach and the more knowledge-intensive approach. In section 3 the experimental set-up for the evaluation is described. The details of the two alternative approaches to explanation (i.e. rule-based and casebased) that have been evaluated are described in sections 4 and 5 . The results of the evaluation are presented in section 6 . The paper finishes with some conclusions and recommendations for future work in section 7 .

\section{Case-Based Explanation}

As stated in the Introduction, our review of the literature suggests that work on CBE can be divided into knowledge-light and knowledge-intensive approaches. However, all approaches to CBR will share an important characteristic. On the spectrum of possibilities between 'specific' and 'general', the case-based explanation will be at the specific end of the spectrum. When discussing explanation patterns (see (Kass \& Leake, 1988) for instance) Kolodner (1996) argues that what differentiates CBR from similar ideas in model-based reasoning is the concreteness of the cases. So, whether knowledge-light or knowledge intensive, case-based explanation is case-based.

There is still disagreement among CBR researchers on the implications CBR has for knowledge engineering effort. Some, such as Mark et al. (1996) argue that CBR still entails a "full knowledge acquisition effort". While others would argue that knowledge-intensive CBR is missing the point of CBR, which is the potential CBR has to finesse knowledge engineering effort by manipulating cases that are compiled chunks of knowledge. These alternative views of CBR are reflected in the different approaches to $\mathrm{CBE}$.

\subsection{Knowledge-Intensive CBE}

A knowledge-intensive approach to CBE will incorporate mechanisms such as rulebased or model-based inference that can be use to generate explanations. Developing knowledge-intensive case-based applications will, in the words of Mark et al (1996), involve a "full scale knowledge acquisition effort". Amongst the earliest examples of this approach are the work on SWALE and its descendants (the CBR systems not the horses). These systems incorporate explanation patterns (XPs) that can be used for explanation. Typically, these XPs are pretty specific, e.g. the JANIS-JOPLIN-XP. Even the more abstract XPs are pretty specific; the MAFIA-REVENGE-XP can be instantiated directly. The key point is that the system designers have incorporated model-based representations that can be used for explanation. 
Another more recent example of a knowledge-intensive approach to CBE is the DIRAS system for assessing long-term risks in diabetes (Armengol et al., 2001). The approach in DIRAS is more dynamic than that in the SWALE systems in the explanation is built at run-time using an a process called Lazy Induction of Descriptions.

\subsection{Knowledge-Light CBE}

The majority of commercially successful CBR applications have been knowledgelight systems; usually retrieval-only systems or mixed initiative systems involving interactive adaptation. In CBR systems that use a feature-value based representation, the retrieved cases can be used in explanation as follows:

"The system predicts that the outcome will be X because that was the

outcome in case $\mathrm{C} 1$ that differed from the current case only in the value of

feature $\mathrm{F}$ which was $\mathrm{f} 2$ instead of $\mathrm{f1}$.

In addition the outcome in C2 was also X ..."

Explanation in these terms (i.e. expressed in the vocabulary of the case features) will not always be adequate but in some situations, such as in medical decision support, it can be quite useful. The main difference between this and the more knowledgeintensive approach described in section 2.1 is that the explanation is expressed in terms of similarity only. The more knowledge-intensive system still produce explanations that reference the retrieved case but the explanation is expressed in terms of causal interactions rather than simple similarity.

A good example of the knowledge-light approach to CBE is the CARES system for predicting recurrence of colorectal cancer developed by Ong, et al. (1997). The approach to explanation in the CARES system is to present the feature-value representations of the retrieved cases and the target case to the user for examination.

The commercial CBR tool Orenge from Empolis* ${ }^{*}$ also highlights this comparison to retrieved cases as a mechanism for explanation.

\section{The Experiment}

Eight unique problem cases were used in the experiment. 37 subjects were presented with each of these problem cases three times, once with predictions and case-based explanations, once with predictions and rule-based explanations (RBE) and once with predictions only without explanation. The rule-based and case-based explanations were presented together but the order was varied to avoid any bias due to familiarity. The format in which the cases and explanations were presented to the user is shown in Fig. 1 and Fig. 2. Fig. 1 shows the case-based explanation while Fig. 2 shows the rule-based explanation.

The subjects were asked to score how convinced they were by the explanations on a 5-point scale (No, Maybe No, Maybe, Maybe Yes, Yes). In the evaluation of the results these scored were interpreted as numeric values from 1-5. The target cases

\footnotetext{
${ }^{*}$ See the White Paper on Orenge available at www.empolis.com.
} 
were presented in turn to the subjects and the subjects were able to backtrack to change their scores.

The subjects were all staff and postgraduate students in the TCD Computer Science Department and it was explained to them that the objective of the experiment was to compare the usefulness of case-based and rule-based explanation.

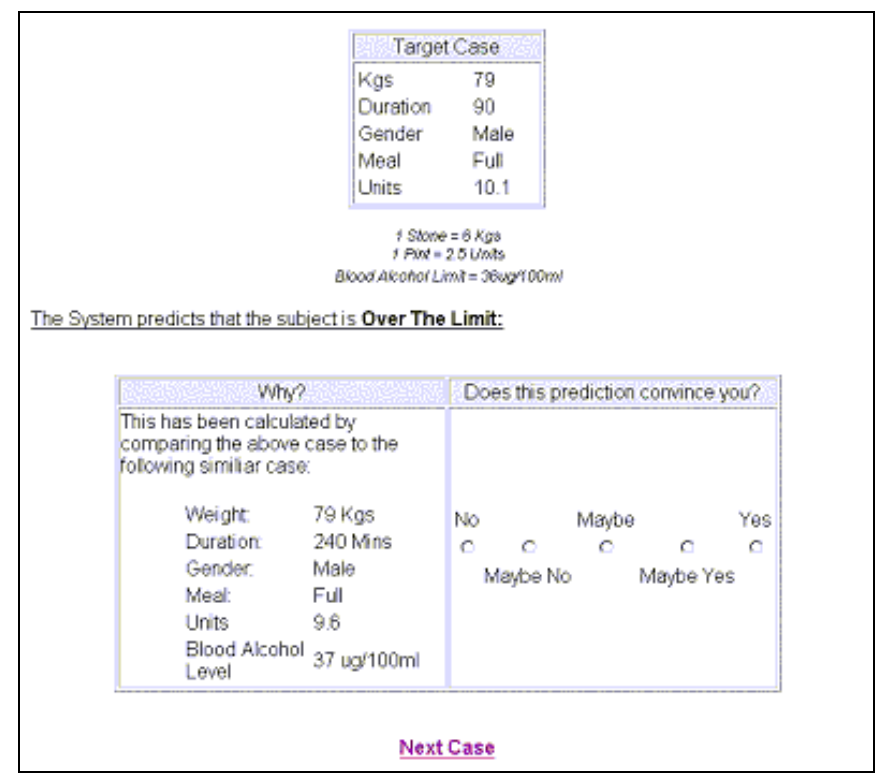

Fig. 1. An example case-based prediction and explanation form the experiment.

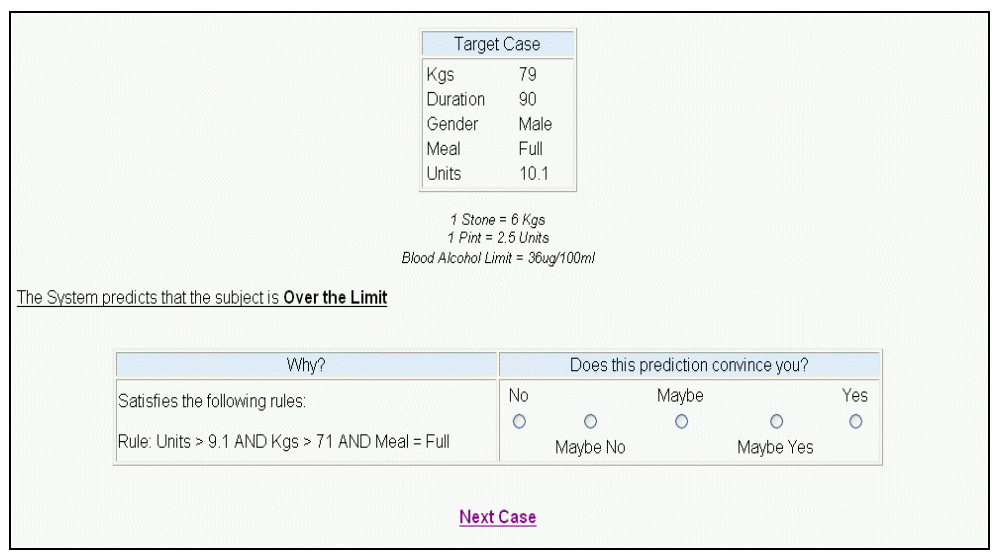

Fig. 2. An example rule-based prediction and explanation from the experiment. 


\section{The Prediction and Explanation Systems}

89 cases were collected in pubs in the centre of Dublin. The alcohol measurements were taken with an Alcho Sensor IV $^{*}$ breath testing system which in an 'evidence grade' system. In addition to the alcohol measurements, the attributes shown in Table 1 were recorded for each case.

Table 1. The features gathered for the experiment.

\begin{tabular}{ll}
\hline Age & Weight \\
Gender & Height \\
Elapsed Time (time since last drink) & Meal (None, Snack, Lunch, Full) \\
Duration (time spent drinking) & Amount (in Units) \\
& Blood Alcohol Content \\
\hline
\end{tabular}

Using a Wrapper-based feature selection technique, we found that using only the features; Weight, Gender, Meal, Duration and Amount produced the best results. Thus the case-based and rule-based prediction and explanation systems were built using 89 cases described by five features. The systems were implemented using the nearest neighbour and decision tree classification code available in the Weka toolkit.**

\subsection{Rule-Based Explanation}

Weka provides the $\mathrm{J} 48$ algorithm, a decision tree-learning algorithm, which is an extension of the C4.5 algorithm (Quinlan, 1993). This code was used to produce the decision tree from which the rules were extracted (see Figure 3). Weka provides code for automatically extracting rules from a decision tree. This code was not used as the rules it produces are designed to be applied in order. Because of this, rules late in the order are incomplete if used as explanations. Instead we extracted complete rules with a comprehensive rule describing each of the possible paths from the root to the leaves of the tree shown in Figure 3. When a new case is passed to the resulting rulebased system for classification, the prediction is produced from the rule that covers it and the rule is also returned as explanation (see Fig. 2). A 10-fold cross validation assessment of the accuracy of the prediction system produced a figure of $80 \%$.

\subsection{Case-Based Explanation}

The Case-Based Explanation system was also developed on top of Weka. Given the feature values for a query case, the system looks at all the existing cases and retrieves the most similar cases from the Case-Base. In the similarity metric used, nominal values such as Gender and Meal simply contribute binary similarity scores.

\footnotetext{
* see www.intox.com

** www.cs.waikato.ac.nz/ml/weka
} 
The accuracy of the Case-Based Prediction system was assessed using a 10 fold cross-validation. Using a single nearest neighbour for prediction yielded an accuracy of $81 \%$. In the evaluation, this nearest neighbour was returned as an explanation of the prediction.

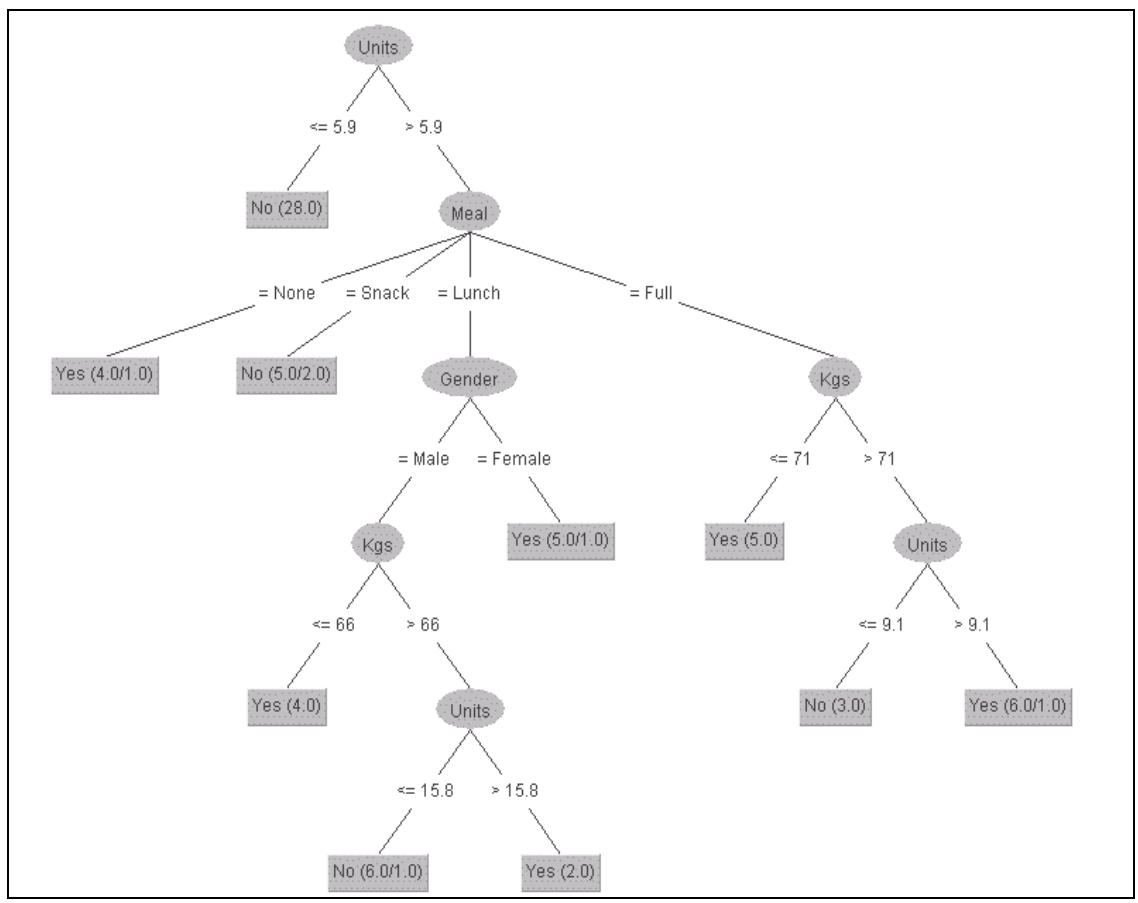

Fig. 3. The decision tree on which the RBE system is built.

\section{Evaluation}

In all 37 subjects evaluated 24 predictions, eight in each category. An incorrect prediction coupled with a poor explanation was included in each category to help assess the attention paid by the subjects to the evaluation. The average rating for these poor predictions was 1.5 while the average for the other predictions was 3.9 (on a scale of 1-5). The ratings for these poor predictions were not considered further in the evaluation. The average of the remaining ratings are shown in Fig. 4. 


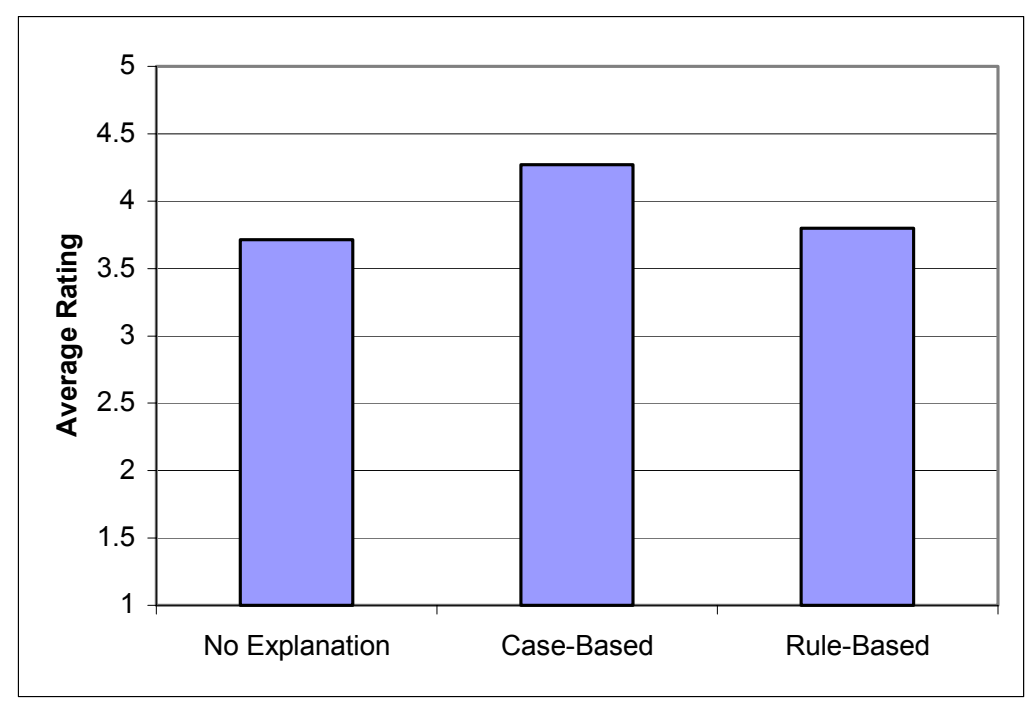

Fig. 4. The average ratings of the three alternative prediction and explanation systems.

Two things to note are the strong performance of the Case-Based explanation and the fact that the predictions without explanation were still found to be quite convincing. Statistical tests were run on the data and a paired t-test showed that the CBE was better than the RBE $(\mathrm{P}$ value $=0.0005)$ and better than No Explanation $(\mathrm{P}$ value $=0.005$ ). If we count the Wins and Draws between the rule-based and casebased alternatives we find that $\mathrm{CBE}$ wins 105 times, RBE wins 48 times and there are 106 draws.

\section{Conclusions and Future Work}

This evaluation provides some support for the use of CBR in applications where explanation of predictions is important. It shows that, in this application area, CBE is considered more convincing than the rule-based alternative. Because of the nature of this type of evaluation it is difficult to perform evaluations across a range of data-sets or domains. So what are the caveats associated with drawing conclusions from this single evaluation?

- Because of the inherent instability of decision tree building algorithms there are alternative decision trees that would have produced different rules that might have scored better.

- CBE may inherently suit this task because it considers all features in the decision making process. The RBE only considers a subset of features and this may be more acceptable in other domains. 
- The comparatively simple case representation may favour CBE. It might fare less well with more complex cases (i.e. more features).

- Results may be different in domains where the subjects have more insight or less insight into the underlying mechanisms.

\subsection{Future Work}

We plan to perform similar evaluations in other domains to explore this question further.

This detailed exploration of the usefulness of knowledge-light CBE suggests ways in which the process might be improved. Comments from evaluators suggest that cases that are perceived to be between the target case and the decision surface are more convincing. For instance, if the target has consumed 10 units and is predicted to be over the limit then a case of 8 units in support of that prediction is more convincing than one of 12 units (other things being equal). It is difficult to select for this using conventional similarity based retrieval. However, order-based retrieval (Bridge 2002) might allow for the selection of more convincing cases.

\section{Acknowledgements}

We would like to thank Ruth Byrne for her advice on the organization of the experiment. We would like to thank Science Foundation Ireland for their support in funding this research.

\section{References}

Armengol, E., Palaudàries, A., Plaza, E., (2001) Individual Prognosis of Diabetes Long-term Risks: A CBR Approach. Methods of Information in Medicine. Special issue in prognostic models in Medicine. vol. 40, pp. 46-51.

Bridge, D., Ferguson, A., (2002) An Expressive Query Language for Product Recommender Systems, Artificial Intelligence Review, vol.18, pp.269-307.

Kass, A.M., Leake, D.B., (1988) Case-Based Reasoning Applied to Constructing Explanations, in Proceedings of 1988 Workshop on Case-Based Reasoning, ed. J. Kolodner, pp190-208, Morgan Kaufmann. San Mateo, Ca.

Kolodner, J., (1996) Making the Implicit Explicit: Clarifying the Principles of Case-Based Reasoning, in Leake, D.B. (ed) Case-Based Reasoning: Experiences, Lessons and Future Directions, pp349-370, MIT Press.

Leake, D., B., (1996) CBR in Context: The Present and Future, in Leake, D.B. (ed) CaseBased Reasoning: Experiences, Lessons and Future Directions, pp3-30, MIT Press.

Mark, W., Simoudis, E., Hinkle, D., (1996) Case-Based Reasoning: Expectations and Results, in Leake, D.B. (ed) Case-Based Reasoning: Experiences, Lessons and Future Directions, pp269-294, MIT Press. 
Ong, L.S., Sheperd, B., Tong, L.C., Seow-Choen, F., Ho, Y.H., Tong, L.C., Ho Y.S, Tan, K. (1997) The Colorectal Cancer Recurrence Support (CARES) System. Artificial Intelligence in Medicine 11(3): 175-188.

Quinlan, J.R., (1993) C4.5: Programs for Machine Learning, Morgan Kaufmann, San Mateo, $\mathrm{Ca}, \mathrm{USA}$.

Riesbeck, C.K., (1988) An Interface for Case-Based Knowledge Acquisition, in Proceedings of 1988 Workshop on Case-Based Reasoning, ed. J. Kolodner, pp312-326, Morgan Kaufmann. San Mateo, Ca. 